\title{
Case report: cognitive performance in an extreme case of anorexia nervosa with a body mass index of 7.7
}

\author{
Simone Daugaard Hemmingsen ${ }^{1,2,3,4,5^{*}}$ (D), Mia Beck Lichtenstein ${ }^{6,7}$ (D), Alia Arif Hussain ${ }^{8,9}$ (D),
} Jan Magnus Sjögren ${ }^{8,9}$ (i) and René Klinkby Støving ${ }^{1,2,3,4}$ (D)

\begin{abstract}
Background: Studies show that adult patients with anorexia nervosa display cognitive impairments. These impairments may be caused by illness-related circumstances such as low weight. However, the question is whether there is a cognitive adaptation to enduring undernutrition in anorexia nervosa. To our knowledge, cognitive performance has not been assessed previously in a patient with anorexia nervosa with a body mass index as low as $7.7 \mathrm{~kg} / \mathrm{m} 2$.

Case presentation: We present the cognitive profile of a 35-year-old woman with severe and enduring anorexia nervosa who was diagnosed at the age of 10 years. She was assessed with a broad neuropsychological test battery three times during a year. Her body mass index was $8.4,9.3$, and $7.7 \mathrm{~kg} / \mathrm{m}^{2}$, respectively. Her general memory performance was above the normal range and she performed well on verbal and design fluency tasks. Her working memory and processing speed were within the normal range. However, her results on cognitive flexibility tasks (set-shifting) were below the normal range.
\end{abstract}

Conclusions: The case study suggests that it is possible to perform normally cognitively despite extreme and chronic malnutrition though set-shifting ability may be affected. This opens for discussion whether patients with anorexia nervosa can maintain neuropsychological performance in spite of extreme underweight and starvation.

Trial registration: ClinicalTrials.gov, NCT02502617. Registered 20 July 2015.

Keywords: Anorexia nervosa, Cognitive performance, Neuropsychology, Emaciation, Undernutrition

\section{Background}

A growing amount of evidence indicate that anorexia nervosa (AN) is associated with impaired or inefficient neuropsychological performance in relation to healthy control subjects, regarding attention [1, 2], memory [1-4], processing speed [4], and especially the executive functions [5] central coherence [6], decision-

\footnotetext{
* Correspondence: simonehemmingsen@gmail.com;

shemmingsen@health.sdu.dk

${ }^{1}$ Centre for Eating Disorder, Odense University Hospital, Odense, Denmark

${ }^{2}$ Research Unit for Medical Endocrinology, Odense University Hospital, Odense, Denmark

Full list of author information is available at the end of the article
}

making [6, 7], and cognitive flexibility [8,9]. It has been debated whether this is related to state (due to factors such as malnutrition) or trait (a premorbid trait or endophenotype of the disorder [10]). Some studies have found that patients who recovered from AN have impaired cognitive performance compared to healthy control subjects $[11,12]$, supporting the trait theory of the disorder. However, longitudinal studies have found that executive functions can be normalized following weight stabilization in patients with AN [13, 14], supporting the state theory.

C C The Author(s). 2020 Open Access This article is licensed under a Creative Commons Attribution 4.0 International License, which permits use, sharing, adaptation, distribution and reproduction in any medium or format, as long as you give appropriate credit to the original author(s) and the source, provide a link to the Creative Commons licence, and indicate if changes were made. The images or other third party material in this article are included in the article's Creative Commons licence, unless indicated otherwise in a credit line to the material. If material is not included in the article's Creative Commons licence and your intended use is not permitted by statutory regulation or exceeds the permitted use, you will need to obtain permission directly from the copyright holder. To view a copy of this licence, visit http://creativecommons.org/licenses/by/4.0/ The Creative Commons Public Domain Dedication waiver (http://creativecommons.org/publicdomain/zero/1.0/) applies to the data made available in this article, unless otherwise stated in a credit line to the data. 
Research on cognitive performance before and after re-nutrition in adult patients with extreme and chronic AN is sparse. Some studies have examined cognitive performance in patients with $\mathrm{AN}$ with a mean body mass index (BMI) below $15 \mathrm{~kg} / \mathrm{m} 2$ (e.g. [10]), corresponding to extreme AN severity according to the Diagnostic and Statistical Manual of Mental Disorders 5 (DSM-5) [15]. However, it is unclear if patients with AN with BMI below $10 \mathrm{~kg} / \mathrm{m} 2$ will display the same cognitive profile.

It has been suggested that malnutrition might affect cognitive performance since the classic Minnesota SemiStarvation Experiment [16], where cognitive functions were studied in 36 healthy military objectors with normal weight before and after semistarvation with $25 \%$ weight-loss over a 24-week period. The men reported decline in concentration. However, the standardized tests that were administered did not confirm measurable alterations. Newer research on healthy subjects, although somewhat inconclusive, indicates affected psychomotor speed and executive functions following short-term semi-starvation [17].

However, other factors than malnutrition or weightloss have been suggested to affect cognitive performance in patients with AN, such as long illness duration [18] and age [18]. This could explain a difference in results for children/adolescents and adults with AN mentioned in the literature $[19,20]$, which cannot be explained by the trait theory.

The current case report was part of an ongoing longitudinal research project investigating the effect of renutrition on cognitive performance in patients with severe $A N$. The aim of the case study was to present the neuropsychological performance of a patient with chronic AN and extremely low BMI in order to discuss whether extremely low weight and long duration of illness are associated with cognitive impairment and if cognitive adaptation takes place. No study to our knowledge has previously reported on the cognitive profile of a patient with AN with a BMI as low as $7.7 \mathrm{~kg} / \mathrm{m} 2$.

We want to introduce the idea of cognitive adaptation to severe malnutrition as a supplement to the discussion on cognitive impairment in AN. However, this idea should not be confused with Taylor's Theory of Cognitive Adaptation [21]. The presented idea of cognitive adaptation is the idea that cognitive functions can adapt to persisting low weight in AN, i.e. cognitive performance can remain normal or regain normality in severe and enduring AN. The adaptation does not exclude specific cognitive impairment.

\section{Case presentation}

The current case report investigates the cognitive profile of a 35-year-old Caucasian woman with extremely severe and enduring $\mathrm{AN}$ who was diagnosed at the age of 10 years. The patient's weight loss is accomplished through fasting. According to the DSM-5 [15], the patient's symptoms are in accordance with the restricting type and the severity of AN for the patient is categorized as extreme. The patient has had low body weight since the onset of the disease 25 years ago. Consequently, she is still prepubescent.

The patient's extreme malnutrition, the medical complications, and the refeeding treatment has previously been described in a case report [22]. Since the previous report [22], she has survived another 5 years, living in her own residence with several stabilizing hospitalizations. Her nadir BMI, defined as the lowest registered BMI, has decreased further to $7.2 \mathrm{~kg} / \mathrm{m} 2$. To our knowledge, this is the lowest BMI reported in AN in the literature. During her long and severe illness course, she has participated in psychotherapy for years. However, during the past few years, she has refused to participate in psychotherapy, while she has continued the harmreducing treatment in the nutrition department. No cognitive profile has been assessed before the current report.

She has continuously been provided supplementation with vitamins and minerals. At the present admission, she weighed $20.2 \mathrm{~kg}$, including edema corresponding to at least $2 \mathrm{~kg}$, and her height was $1.55 \mathrm{~m}$, corresponding to a BMI of $8.41 \mathrm{~kg} / \mathrm{m}^{2}$. After life-saving and stabilizing fluid and electrolyte correction, and refeeding according to guidelines [23] during 2 weeks of hospitalization, we tested her with a neuropsychological test battery (2 weeks after admission: $\mathrm{T}_{0}$ ). After an additional 2 months of hospitalization, she could not be motivated to continue the treatment any longer. Due to years of history with rapid relapse after prolonged forced treatment, she was allowed to be discharged to outpatient follow-up. She was re-tested in the outpatient clinic 6 days following dropout from inpatient treatment and approximately 3 months after admission, (re-test: $\mathrm{T}_{1}$ ) with a weight of $22.4 \mathrm{~kg}$ (BMI: $9.3 \mathrm{~kg} / \mathrm{m}^{2}$ ), and again at 12 months from $\mathrm{T}_{0}$, during a re-hospitalization, 7 days after admission (follow-up: $\mathrm{T}_{2}$ ), with BMI $7.7 \mathrm{~kg} / \mathrm{m}^{2}$. Thus, $\mathrm{T}_{0}$ and $\mathrm{T}_{2}$ were done at the hospital after initial stabilizing glycemic, fluid- and electrolyte correction, whereas $T_{1}$ was done in an outpatient setting, where she was in a clinically stable condition, but without the initial stabilizing treatment.

\section{The psychopathological profile of the patient}

The patient scored 21 on the Beck Depression Inventory II (BDI-II [24];) indicating moderate depression at 2 weeks after admission $\left(\mathrm{T}_{0}\right)$. Her scores on the Eating Disorder Inventory 3 (EDI-3 [25];) at $\mathrm{T}_{0}$ are presented in Table 1 below. Compared to the Danish validation of EDI-3 for patients with AN ( [26]; Table 1), her low 
Table 1 Eating Disorder Inventory 3 (EDI-3) scores for the patient and from the Danish validation of EDI- $3^{\mathrm{a}}$ on a sample of patients with anorexia nervosa (AN)

\begin{tabular}{lll}
\hline EDI-3 subscales & $\begin{array}{l}\text { Scores in the } \\
\text { present case } \\
\text { of AN }\end{array}$ & $\begin{array}{l}\text { Danish validation } \\
\text { sample of AN } \\
(\boldsymbol{N}=84)^{\mathbf{a}} \text { Mean (SD) }\end{array}$ \\
\hline Drive for thinness (DT) & 7 & $18.7(6.3)$ \\
Bulimia (B) & 0 & $8.0(8.0)$ \\
Body dissatisfaction (BD) & 18 & $24.4(9.3)$ \\
Low self-esteem (LSE) & 21 & $13.8(5.6)$ \\
Personal alienation (PA) & 10 & $13.1(5.3)$ \\
Interpersonal insecurity (II) & 10 & $10.0(5.6)$ \\
Interpersonal alienation (IA) & 5 & $9.3(5.4)$ \\
Interoceptive deficits (ID) & 2 & $19.9(7.6)$ \\
Emotional dysregulation (ED) & 3 & $8.9(5.1)$ \\
Perfectionism (P) & 1 & $11.0(4.6)$ \\
Asceticism (AS) & 1 & $12.9(6.0)$ \\
Maturity fear (MF) & 9 & $12.2(6.9)$ \\
\hline
\end{tabular}

${ }^{\mathrm{a} C l a u s e n ~ e t ~ a l . ~[26] ~}$

scores on the Drive for Thinness, the Interoceptive Deficits, the Perfectionism, and the Asceticism subscales are of interest.

\section{Qualitative observations}

During the first 2 weeks after admission, the patient was unable to participate in the neuropsychological assessment due to fatigue. Two weeks after admission, when the baseline assessment took place $\left(\mathrm{T}_{0}\right)$, the patient was lying down during the assessment and was noticeably tired. This was neither the case at retest $\left(T_{1}\right)$ nor at follow-up $\left(\mathrm{T}_{2}\right)$ where the patient was sitting at a table. Her alertness and energy level at follow-up $\left(T_{2}\right)$ were notable in light of her low BMI. The patient was calm during all three assessments (divided into six sessions) and expressed that the tests were fun. The aim of the study was explained to the patient before the first administration. However, only information written in the test manuals was given during each assessment.

\section{Measures}

The following validated neuropsychological tests were selected in cooperation with an experienced neuropsychologist to examine a wide range of cognitive functions: the Wechsler Memory Scale III (WMS-III) [27]; the d2$\mathrm{R}$ Test of Attention - Revised [28, 29]; the Processing Speed Index (PSI) of the Wechsler Adult Intelligence Scale IV (WAIS-IV) [30]; the Delis-Kaplan Executive Function System (D-KEFS) [31], Verbal Fluency Test, Design Fluency Test and Trail Making Test; and the Wisconsin Card Sorting Test Revised and Expanded (WCST) [32] (only administered at $\mathrm{T}_{0}$ ). Information on each test variable, including internal consistency and test-retest reliability, are presented in Table 2 . The test battery can be administered in approximately $2 \mathrm{~h}$. For all three administrations, the test battery was divided into two sessions (1 h per session) 1 day apart.

\section{Neuropsychological findings}

Table 3 gives an overview of the timeline of the patient's raw scores and scaled scores on the test battery. Table 4 presents the patient's norm scores and percentiles on the WMS-III, the WAIS-IV PSI, and the d2-R. Table 5 presents the patient's WCST scores at 2 weeks after admission $\left(\mathrm{T}_{0}\right)$. Information on scoring are presented below each of the tables.

\section{Memory performance on WMS-III}

The patient's scores on WMS-III indicate average to very superior auditory, visual, immediate and general memory performance (108 to 142; Mean: 100), and low average to average working memory (Table 4). The technical manual for WMS-III reports adequate test - retest reliability for all indexes in the age group 16-54 years, except for the Auditory Recognition Delayed Index ( [33]; Table 2). Estimated standard error of difference $\left(\mathrm{S}_{\text {Diff }}\right)$ scores were calculated based on Iverson and Grant ( [34]; Table 2). Differences between the three assessments are outlined here. Her scores on the Auditory Delayed Index decreased more than $S_{\text {diff: }} 6.70$ from 132 (very superior) at 2 weeks after admission $\left(\mathrm{T}_{0}\right)$ to 108 (average) at re-test $\left(\mathrm{T}_{1}\right)$ and increased again to 132 (very superior) at follow-up $\left(\mathrm{T}_{2}\right)$. Her scores on the Visual Immediate Index increased slightly more than $S_{\text {diff: }} 6.70$ from 118 (high average) at re-test $\left(\mathrm{T}_{1}\right)$ to 127 (superior) at follow-up $\left(\mathrm{T}_{2}\right)$. Her scores on the Visual Delayed Index decreased more than $S_{\text {diff: }} 7.65$ from 125 (superior) at re-test $\left(\mathrm{T}_{1}\right)$ to 109 (average) at follow-up $\left(\mathrm{T}_{2}\right)$. Her scores on the Immediate Memory Index increased more than $S_{\text {diff: }} 3.17$ from 134 (very superior) at re-test $\left(\mathrm{T}_{1}\right)$ to 142 (very superior) at follow-up $\left(\mathrm{T}_{2}\right)$. Her scores on the Working Memory Index decreased more than $S_{\text {diff: }} 8.22$ from 102 (average) at 2 weeks after admission $\left(\mathrm{T}_{0}\right)$ to 88 (low average) at re-test $\left(\mathrm{T}_{1}\right)$. The scores on the rest of the indexes did not change more than the estimated $\mathrm{S}_{\text {diff }}$ scores between time points.

\section{Cognitive flexibility on D-KEFS and WCST}

Overall, she performed above average on the Verbal Fluency Test (Table 3) at all three test times compared to the normative population for age, except for her performance at re-test $\left(\mathrm{T}_{1}\right)$ on the switching condition, which was decreased more than $S_{\text {diff: }} 2.42$ to average, and the high number of repetition errors (7; below average) at re-test $\left(\mathrm{T}_{1}\right)$ and (3; average) at follow-up $\left(\mathrm{T}_{2}\right)$.

She performed average to above average on the Design Fluency Test at all three test sessions (Table 3). 
Table 2 The neuropsychological test battery

\begin{tabular}{|c|c|c|c|c|}
\hline Instrument & Variables & Description & $\begin{array}{l}\text { Higher/lower scores } \\
\text { indicating improvement }\end{array}$ & Reliability \\
\hline \multirow[t]{11}{*}{ Wechsler Memory Scale III (WMS-III) $)^{1}$} & Logical memory I & Task: History recitation & Higher & $\begin{array}{l}\text { Adequate internal } \\
\text { consistency: } 0.88^{\mathrm{a}} \\
\text { Adequate test - } \\
\text { retest: } 0.77^{\mathrm{b}} \\
\mathrm{SE}_{\mathrm{M}: 1.04^{\mathrm{a}}} \\
\mathrm{S}_{\text {diff. }} 1.47\end{array}$ \\
\hline & Logical Memory II & Task: Delayed history recitation & Higher & $\begin{array}{l}\text { Low internal } \\
\text { consistency: } \\
0.84^{\mathrm{a}} \\
\text { Adequate test - } \\
\text { retest: } 0.77^{\mathrm{b}} \\
\mathrm{SE}_{\mathrm{M}}: 1.20^{\mathrm{a}} \\
\mathrm{S}_{\text {diff: }} 1.70\end{array}$ \\
\hline & $\begin{array}{l}\text { Verbal Paired } \\
\text { Associates I }\end{array}$ & Task: Word pairing & Higher & $\begin{array}{l}\text { Adequate internal } \\
\text { consistency: } 0.95^{\mathrm{a}} \\
\text { Adequate test - } \\
\text { retest: } 0.77^{\mathrm{b}} \\
\mathrm{SE}_{\mathrm{M}}: 0.67^{\mathrm{a}} \\
\mathrm{S}_{\text {diff: }} 0.95\end{array}$ \\
\hline & $\begin{array}{l}\text { Verbal Paired } \\
\text { Associates II }\end{array}$ & Task: Delayed word pairing & Higher & $\begin{array}{l}\text { Adequate internal } \\
\text { consistency: } 0.88^{\mathrm{a}} \\
\text { Adequate test - } \\
\text { retest: } 0.73^{\mathrm{b}} \\
\mathrm{SE}_{\mathrm{M}}: 1.04^{\mathrm{a}} \\
\mathrm{S}_{\text {diff: }} \cdot 1.47\end{array}$ \\
\hline & Faces I & $\begin{array}{l}\text { Task: Face recognition among other } \\
\text { faces }\end{array}$ & Higher & $\begin{array}{l}\text { Low internal } \\
\text { consistency: } \\
0.77^{\mathrm{a}} \\
\text { Low test - retest: } \\
0.64^{\mathrm{b}} \\
\mathrm{SE}_{\mathrm{M}}: 1.44^{\mathrm{a}} \\
\mathrm{S}_{\text {diff: }} 2.04\end{array}$ \\
\hline & Faces $\|$ & Task: Delayed face recognition & Higher & $\begin{array}{l}\text { Low internal } \\
\text { consistency: } 0.83^{\mathrm{a}} \\
\text { Low test - retest: } \\
0.58^{\mathrm{b}} \\
\mathrm{SE}_{\mathrm{M}}: 1.24^{\mathrm{a}} \\
\mathrm{S}_{\text {diff: }} 1.75\end{array}$ \\
\hline & Family Pictures I & Task: Picture recall & Higher & $\begin{array}{l}\text { Adequate internal } \\
\text { consistency: } 0.86^{\mathrm{a}} \\
\text { Low test - retest: } \\
0.61^{\mathrm{b}} \\
\mathrm{SE}_{\mathrm{M}}: 1.12^{\mathrm{a}} \\
\mathrm{S}_{\text {diff: }} \cdot 1.58\end{array}$ \\
\hline & Family Pictures II & Task: Delayed picture recall & Higher & $\begin{array}{l}\text { Adequate internal } \\
\text { consistency: } 0.85^{\mathrm{a}} \\
\text { Low test - retest: } \\
0.67^{\mathrm{b}} \\
\mathrm{SE}_{\mathrm{M}}: 1.16^{\mathrm{a}} \\
\mathrm{S}_{\text {diff: }} 1.64\end{array}$ \\
\hline & $\begin{array}{l}\text { Letter-Number } \\
\text { Sequencing }\end{array}$ & $\begin{array}{l}\text { Task: Recall and ordering letters and } \\
\text { numbers }\end{array}$ & Higher & $\begin{array}{l}\text { Low internal } \\
\text { consistency: } \\
0.75^{\mathrm{a}} \\
\text { Low test - retest: } \\
0.61^{\mathrm{b}} \\
\mathrm{SE}_{\mathrm{M}}: 1.50^{\mathrm{a}} \\
\mathrm{S}_{\text {diff: }} 2.12\end{array}$ \\
\hline & Spatial Span & Task: Visual pointing recall & Higher & $\begin{array}{l}\text { Low internal } \\
\text { consistency: } \\
0.82^{\mathrm{a}} \\
\text { Low test - retest: } \\
0.65^{\mathrm{b}} \\
\mathrm{SE}_{\mathrm{M}}: 1.27^{\mathrm{a}} \\
\mathrm{S}_{\text {diff: }} 1.80\end{array}$ \\
\hline & Auditory Immediate & Index: Measuring auditory immediate & Higher & Adequate internal \\
\hline
\end{tabular}


Table 2 The neuropsychological test battery (Continued)

\begin{tabular}{|c|c|c|c|c|}
\hline Instrument & Variables & Description & $\begin{array}{l}\text { Higher/lower scores } \\
\text { indicating improvement }\end{array}$ & Reliability \\
\hline & Index & memory & & $\begin{array}{l}\text { consistency: } 0.93^{\mathrm{a}} \\
\text { Adequate test - } \\
\text { retest: } 0.84^{\mathrm{b}} \\
\mathrm{SE}_{\mathrm{M}}: 3.97^{\mathrm{a}} \\
\mathrm{S}_{\text {diff: }} .61\end{array}$ \\
\hline & $\begin{array}{l}\text { Auditory Delayed } \\
\text { Index }\end{array}$ & $\begin{array}{l}\text { Index: Measuring auditory delayed } \\
\text { memory }\end{array}$ & Higher & $\begin{array}{l}\text { Adequate internal } \\
\text { consistency: } 0.90^{\mathrm{a}} \\
\text { Adequate test - } \\
\text { retest: } 0.82^{\mathrm{b}} \\
\mathrm{SE}_{\mathrm{M}}: 4.74^{\mathrm{a}} \\
\mathrm{S}_{\text {diff: }} 6.70\end{array}$ \\
\hline & $\begin{array}{l}\text { Visual Immediate } \\
\text { Index }\end{array}$ & $\begin{array}{l}\text { Index: Measuring visual immediate } \\
\text { memory }\end{array}$ & Higher & $\begin{array}{l}\text { Adequate internal } \\
\text { consistency: } 0.85^{\mathrm{a}} \\
\text { Adequate test - } \\
\text { retest: } 0.74^{\mathrm{b}} \\
\mathrm{SE}_{\mathrm{M}}: 5.81^{\mathrm{a}} \\
\mathrm{S}_{\text {diff: }} 8.22\end{array}$ \\
\hline & Visual Delayed Index & $\begin{array}{l}\text { Index: Measuring visual delayed } \\
\text { memory }\end{array}$ & Higher & $\begin{array}{l}\text { Adequate internal } \\
\text { consistency: } 0.87^{\mathrm{a}} \\
\text { Adequate test - } \\
\text { retest: } 0.74^{\mathrm{b}} \\
\mathrm{SE}_{\mathrm{M}}: 5.41^{\mathrm{a}} \\
\mathrm{S}_{\text {diff: }} .65\end{array}$ \\
\hline & $\begin{array}{l}\text { Immediate Memory } \\
\text { index }\end{array}$ & Index: Measuring immediate memory & Higher & $\begin{array}{l}\text { Adequate internal } \\
\text { consistency: } 0.92^{\mathrm{a}} \\
\text { Adequate test - } \\
\text { retest: } 0.84^{\mathrm{b}} \\
\mathrm{SE}_{\mathrm{M}}: 4.24^{\mathrm{a}} \\
\mathrm{S}_{\text {diff: }} 3.17\end{array}$ \\
\hline & $\begin{array}{l}\text { Auditory Recognition } \\
\text { Delayed Index }\end{array}$ & Index: Measuring recognition & Higher & $\begin{array}{l}\text { Low internal } \\
\text { consistency: } \\
0.74^{\mathrm{a}} \\
\text { Low test - retest: } \\
0.60^{\mathrm{b}} \\
\mathrm{SE}_{\mathrm{M}}: 7.65^{\mathrm{a}} \\
\mathrm{S}_{\text {diff: }} 10.82\end{array}$ \\
\hline & $\begin{array}{l}\text { General Memory } \\
\text { Index }\end{array}$ & $\begin{array}{l}\text { Index: A global measure of delayed } \\
\text { memory }\end{array}$ & Higher & $\begin{array}{l}\text { Adequate internal } \\
\text { consistency: } 0.93^{\mathrm{a}} \\
\text { Adequate test - } \\
\text { retest: } 0.87^{\mathrm{b}} \\
\mathrm{SE}_{\mathrm{M}}: 3.97^{\mathrm{a}} \\
\mathrm{S}_{\text {diff: }} 5.61\end{array}$ \\
\hline & $\begin{array}{l}\text { Working Memory } \\
\text { Index }\end{array}$ & Index: Measuring working memory & Higher & $\begin{array}{l}\text { Adequate internal } \\
\text { consistency: } 0.85^{\mathrm{a}} \\
\text { Adequate test - } \\
\text { retest: } 0.70^{\mathrm{b}} \\
\mathrm{SE}_{\mathrm{M}}: 5.81^{\mathrm{a}} \\
\mathrm{S}_{\text {diff: }} 8.22\end{array}$ \\
\hline \multirow[t]{5}{*}{$\begin{array}{l}\text { Processing Speed Index (PSI) of the Wechsler } \\
\text { Adult Intelligence Scale IV (WAIS-IV) }\end{array}$} & Symbol Search & $\begin{array}{l}\text { Task: Search for symbols in rows of } \\
\text { symbols }\end{array}$ & Higher & $\begin{array}{l}\text { Low internal } \\
\text { consistency: } 0.73^{\mathrm{a}} \\
\text { Adequate test - } \\
\text { retest: } 0.75^{\mathrm{c}} \\
\mathrm{SE}_{\mathrm{M}}: 1.56^{\mathrm{a}} \\
\mathrm{S}_{\text {diff: }} \cdot 2.21\end{array}$ \\
\hline & Symbol Search errors & - & Lower & - \\
\hline & Coding & $\begin{array}{l}\text { Task: Write correct symbols under } \\
\text { numbers }\end{array}$ & Higher & $\begin{array}{l}\text { Internal } \\
\text { consistency: } 0.84^{\mathrm{a}} \\
\text { Adequate test - } \\
\text { retest: } 0.83^{\mathrm{C}} \\
\mathrm{SE}_{\mathrm{M}}: 1.20^{\mathrm{a}} \\
\mathrm{S}_{\text {diff: }} 1.70\end{array}$ \\
\hline & Coding errors & - & Lower & - \\
\hline & $\begin{array}{l}\text { Processing Speed } \\
\text { Index }\end{array}$ & $\begin{array}{l}\text { Index: Measuring processing speed } \\
\text { (and attention) }\end{array}$ & Higher & $\begin{array}{l}\text { Adequate internal } \\
\text { consistency: }\end{array}$ \\
\hline
\end{tabular}


Table 2 The neuropsychological test battery (Continued)

\begin{tabular}{lll}
\hline Instrument & Variables & Description \\
\hline d2-R Test of Attention - Revised ${ }^{3}$ & Processed Targets & $\begin{array}{l}\text { Task: Search for d's with marks in rows } \\
\text { of letters with marks }\end{array}$ \\
& $\begin{array}{l}\text { Errors } \\
\text { Accuracy (\% errors) }\end{array}$ & - \\
Corrected total score & $\begin{array}{l}\text { Measuring attention, (and processing } \\
\text { speed and response inhibition) }\end{array}$ \\
& & \\
& $\begin{array}{l}\text { Concentration } \\
\text { Performance }\end{array}$ & Measuring concentration
\end{tabular}

\begin{tabular}{|c|c|}
\hline Higher/lower scores & Reliability \\
\hline & $\begin{array}{l}0.87^{\mathrm{a}} \\
\text { Adequate test - } \\
\text { retest: } 0.87^{\mathrm{C}} \\
\mathrm{SE}_{\mathrm{M}}: 5.41^{\mathrm{a}} \\
\mathrm{S}_{\text {diff. }} 7.65\end{array}$ \\
\hline Higher & - \\
\hline Lower & - \\
\hline Lower & - \\
\hline Higher & $\begin{array}{l}\text { High test - retest: } \\
0.87 \\
\text { SE }_{M}: 40.1^{d} \\
\text { S }_{\text {diff: }} 56.71\end{array}$ \\
\hline Higher & $\begin{array}{l}\text { High test - retest: } \\
0.91 \\
\mathrm{SE}_{\mathrm{M}}: 17.6^{\mathrm{d}} \\
\mathrm{S}_{\text {diff: }} \cdot 24.89\end{array}$ \\
\hline
\end{tabular}

Delis-Kaplan Executive Function System (DKEFS) $)^{4}$ :

Verbal Fluency Test

\section{Condition 1: Phonemic/ Letter Fluency}

Task: Words with specific first letter Measuring word fluency

Task: Animals and boy's names Semantic/ Category Measuring word fluency Fluency

Condition 3: Category Switching

Task: Switch between furniture and fruits Measuring cognitive flexibility

Design Fluency Test

Trail Making Test

Category Errors

Condition 1: Filled

Dot

Task: Draw different designs

Measuring design fluency

Condition 2: Empty

Dots Only the use of filled dots

Measuring design fluency and response inhibition

Condition 3:

Switching dots

Measuring cognitive flexibility

Repetition Errors

Category Errors

Condition 1: Visual

$-$

Task: Cross 3's among numbers and
Task: Draw different designs without

Task: Switch between filled and empty

Higher

Higher

High internal

consistency: $0.90^{\circ}$

Moderate test -

retest: $0.76^{\mathrm{f}}$

$\mathrm{SE}_{\mathrm{M}}: 0.97^{\mathrm{e}}$

$S_{\text {diff: }} 1.37$

Higher

Moderate internal

consistency: $0.76^{\mathrm{e}}$

Good test - retest:

$0.81^{\mathrm{f}}$

$\mathrm{SE}_{\mathrm{M}}: 1.48^{\mathrm{e}}$

$S_{\text {diff: }} 2.09$

Higher

Moderate internal consistency: $0.68^{\mathrm{e}}$ Low test - retest: $0.49^{f}$

$\mathrm{SE}_{\mathrm{M}}: 1.71^{\mathrm{e}}$

$\mathrm{S}_{\text {diff: }} 2.42$

Lower

Lower

Higher

Moderate test -

retest: $0.62^{f}$

$\mathrm{SE}_{\mathrm{M}}: 1.94^{\mathrm{g}}$

$S_{\text {diff: }} 2.74$

Higher

Moderate test -

retest: $0.73^{f}$

$\mathrm{SE}_{\mathrm{M}}: 1.98^{\mathrm{g}}$

$S_{\text {diff: }} 2.80$

Low test - retest:

$0.22^{f}$

$\mathrm{SE}_{\mathrm{M}}: 2.47^{9}$

$S_{\text {diff: }} 3.49$

search

Lower

Lower letters

Measuring attention, and processing speed

Lower

$-$

$-$

Low test - retest: $0.55^{f}$

Lower

Low test - retest: $0.54^{f}$

Condition 2: Numbers Task: Draw line between numbers in order among numbers and letters Measuring attention and processing speed 
Table 2 The neuropsychological test battery (Continued)

\begin{tabular}{|c|c|c|c|c|}
\hline Instrument & Variables & Description & $\begin{array}{l}\text { Higher/lower scores } \\
\text { indicating improvement }\end{array}$ & Reliability \\
\hline & Condition 3: Letters & $\begin{array}{l}\text { Task: Draw line between letters in } \\
\text { order among numbers and letters } \\
\text { Measuring attention and processing } \\
\text { speed }\end{array}$ & Lower & $\begin{array}{l}\text { Low test - retest: } \\
0.48^{f}\end{array}$ \\
\hline & $\begin{array}{l}\text { Condition 4: Number- } \\
\text { Letter }\end{array}$ & $\begin{array}{l}\text { Task: Switch between numbers and } \\
\text { letters } \\
\text { Measuring cognitive flexibility }\end{array}$ & Lower & $\begin{array}{l}\text { Low test - retest: } \\
0.36^{f}\end{array}$ \\
\hline & Condition 4 Errors & - & Lower & - \\
\hline & $\begin{array}{l}\text { Condition 5: Motor } \\
\text { Speed }\end{array}$ & - & Lower & $\begin{array}{l}\text { Moderate test - } \\
\text { retest: } 0.73^{f}\end{array}$ \\
\hline & $\begin{array}{l}\text { Combined Number + } \\
\text { Letter Sequencing }\end{array}$ & - & Lower & $\begin{array}{l}\text { Moderate internal } \\
\text { consistency: } 0.78^{\mathrm{e}} \\
\text { Moderate test - } \\
\text { retest: } 0.64^{\mathrm{f}} \\
\mathrm{SE}_{\mathrm{M}}: 1.41^{\mathrm{g}} \\
\mathrm{S}_{\text {diff: }} 1.99\end{array}$ \\
\hline \multirow[t]{9}{*}{$\begin{array}{l}\text { Wisconsin Card Sorting Test Revised and } \\
\text { Expanded (WCST) }\end{array}$} & Trials Administered & $\begin{array}{l}\text { Task: Matching cards to key cards } \\
\text { based on color, form, or number }\end{array}$ & Lower & - \\
\hline & Correct trials & - & Higher & - \\
\hline & Errors & - & Lower & $\begin{array}{l}\text { Generalizability } \\
\text { coefficient: } 0.71 \\
\mathrm{SE}_{\mathrm{M}}: 8.08\end{array}$ \\
\hline & $\begin{array}{l}\text { Perseverative } \\
\text { Responses }\end{array}$ & Measuring cognitive inflexibility & Lower & $\begin{array}{l}\text { Generalizability } \\
\text { coefficient: } 0.53 \\
\text { SE }_{M}: 10.28\end{array}$ \\
\hline & Perseverative Errors & Measuring cognitive inflexibility & Lower & $\begin{array}{l}\text { Generalizability } \\
\text { coefficient: } 0.52 \\
\text { SE }_{M}: 10.39\end{array}$ \\
\hline & $\begin{array}{l}\text { Non-perseverative } \\
\text { Errors }\end{array}$ & - & Lower & $\begin{array}{l}\text { Generalizability } \\
\text { coefficient: } 0.72 \\
\text { SE }_{M}: 7.94\end{array}$ \\
\hline & $\begin{array}{l}\text { Conceptual Level } \\
\text { Responses }\end{array}$ & - & Higher & - \\
\hline & Categories Completed & - & Higher & - \\
\hline & Failure to Maintain Set & - & Lower & - \\
\hline
\end{tabular}

\footnotetext{
${ }^{1}$ Information from WAIS-III WMS-III Technical Manual (2002), re-test interval WMS-III = 35.6 days; ${ }^{2}$ Information from WAIS-IV Technical and Interpretive Manual (2008), re-test interval WAIS-IV = 22 days; ${ }^{3}$ Information from d2 Test of Attention in Danish d2-testen - en vurdering af opmærksomhed of koncentration (2006), re-test interval d2 test $=8-9$ weeks; ${ }^{4}$ Information from D-KEFS Technical Manual (2001), re-test interval D-KEFS $=25$ days; ${ }^{5}$ Information from Wisconsin Card Sorting Test Manual - Revised and Expanded (1993), re-test interval WCST = 32.61 days $S E_{M}$ Standard errors of measurement (reported in scaled-score units for subtests (for WMS-III, WAIS_IV, and D-KEFS), in index units for index scores (for WMS and WAIS), and in total score units (for d2 test and WCST))

$S_{\text {diff }}$ Estimated standard error of the difference scores (calculated based on Iverson and Grant (2001))

${ }^{a}$ Reported in relation to age $35-44$ years; ${ }^{\text {b }}$ Reported in relation to age $16-54$ years; ${ }^{\text {c }}$ Reported in relation to age $30-54$ years; ${ }^{d}$ Reported for whole sample 20-60 years; ${ }^{\mathrm{e}}$ Reported in relation to age 30-39 years; ${ }^{\mathrm{f}}$ Reported in relation to age $20-49$ years; ${ }^{9}$ Reported for whole sample $8-89$ years
}

However, the switching condition score was lower [6] at follow-up $\left(\mathrm{T}_{2}\right)$ compared to 8 at 2 weeks after admission $\left(\mathrm{T}_{0}\right)$ and re-test $\left(\mathrm{T}_{1}\right)$, though still average.

During follow-up $\left(\mathrm{T}_{2}\right)$ on the Trail Making Test (Table 3), her performance on the Number-Letter Sequencing test, measuring cognitive flexibility, was below average (111 s), in spite of being average at 2 weeks after admission $\left(\mathrm{T}_{0} ; 90 \mathrm{~s}\right)$ and re-test $\left(\mathrm{T}_{1} ; 79 \mathrm{~s}\right)$. The numbers condition was very low at $\mathrm{T}_{0}(55 \mathrm{~s}$; below average), improving somewhat at re-test $\left(\mathrm{T}_{1}\right.$; $46 \mathrm{~s}$; below average) and follow-up $\left(\mathrm{T}_{3} ; 41 \mathrm{~s}\right.$; below average). We have no explanation for this result. On the other conditions, her performance was average at all three test times on the Trail Making Test.

Her scores on the WCST (Table 5) 2 weeks after admission $\left(\mathrm{T}_{0}\right)$ place her in the mild to moderately-to-severely range of impairment on cognitive flexibility according to this task. She completed one out of six categories $(<1$ st percentile). She made 52 perseverative responses $(<1$ st percentile; standard score 55; moderately-to-severely impaired range). She committed 50 errors (8th percentile; standard score 79: mildly impaired range), of which 36 were perseverative errors (1st percentile; standard score 55: moderately impaired range). 
Table 3 The patient's neuropsychological performance raw scores and scaled scores at two weeks after admission $\left(T_{0}\right)$, at re-test $\left(T_{1}\right)$, and at follow-up $\left(T_{2}\right)$

\begin{tabular}{|c|c|c|c|c|c|c|}
\hline \multirow[t]{2}{*}{ Instrument } & \multicolumn{3}{|c|}{ Raw scores } & \multicolumn{3}{|c|}{ Scaled scores } \\
\hline & $\mathrm{T}_{0}$ & $\mathrm{~T}_{1}$ & $\mathrm{~T}_{2}$ & $\mathrm{~T}_{0}$ & $\mathrm{~T}_{1}$ & $\mathrm{~T}_{2}$ \\
\hline \multicolumn{7}{|l|}{ WMS-III } \\
\hline Logical Memory I & 62 & 61 & 66 & 16 & 16 & 17 \\
\hline Logical Memory ॥ & 42 & 46 & 43 & 17 & 18 & 17 \\
\hline Verbal Paired Associated I & 32 & 32 & 32 & 16 & 16 & 16 \\
\hline Verbal Paired Associated II & 8 & 8 & 8 & 13 & 13 & 13 \\
\hline Faces I & 42 & 41 & 45 & 12 & 11 & 15 \\
\hline Faces II & 44 & 42 & 45 & 14 & 12 & 15 \\
\hline Family Pictures I & 56 & 62 & 59 & 13 & 15 & 14 \\
\hline Family Pictures ॥ & 56 & 62 & 39 & 12 & 16 & 8 \\
\hline Letter-Number Sequencing & 11 & 9 & 10 & 10 & 8 & 9 \\
\hline Spatial Span & 18 & 13 & 16 & 11 & 8 & 10 \\
\hline \multicolumn{7}{|l|}{ d2-R } \\
\hline Processed Targets & 426 & 420 & 451 & & & \\
\hline Errors & 3 & 0 & 4 & & & \\
\hline Accuracy (\% errors) & 0.70 & 0.00 & 0.89 & & & \\
\hline Corrected total score & 423 & 420 & 447 & & & \\
\hline Concentration Performance & 175 & 176 & 185 & & & \\
\hline \multicolumn{7}{|l|}{ WAIS-IV Processing Speed } \\
\hline Symbol Search & 28 & 28 & 35 & 7 & 8 & 10 \\
\hline Symbol Search Errors & 3 & 0 & 1 & & & \\
\hline Coding & 65 & 63 & 59 & 10 & 9 & 9 \\
\hline Coding Errors & 0 & 0 & 1 & & & \\
\hline D-KEFS & & & & & & \\
\hline
\end{tabular}

\section{Verbal Fluency Test:}

\begin{tabular}{lllllll} 
Condition 1: Phonemic Fluency & 49 & 60 & 56 & 13 & 16 & 15 \\
Condition 2: Semantic Fluency & 58 & 55 & 62 & 17 & 16 & 19 \\
Condition 3: Category Switching & 17 & 14 & 16 & 14 & 10 & 12 \\
Repetition Errors & 2 & 7 & 3 & 10 & 4 & 9 \\
Category Errors & 0 & 0 & 1 & 13 & 13 & 11 \\
Design Fluency Test & & & & & & \\
Condition 1: Filled Dot & 10 & 14 & 15 & 10 & 13 & 14 \\
Condition 2: Empty Dots Only & 15 & 12 & 13 & 13 & 11 & 12 \\
Condition 3: Switching & 8 & 8 & 6 & 10 & 10 & 8 \\
Repetition Errors & 0 & 4 & 3 & 13 & 11 & 12 \\
Category Errors & 2 & 2 & 3 & 12 & 12 & 11 \\
Trail Making Test & & & & & & \\
Condition 1: Visual search & 23 & 21 & 19 & 9 & 10 & 11 \\
Condition 2: Numbers & 55 & 46 & 41 & 3 & 6 & 7 \\
Condition 3: Letters & 32 & 30 & 36 & 10 & 10 & 9 \\
Condition 4: Number-Letter & 90 & 79 & 111 & 9 & 10 & 6 \\
Condition 4 Errors & 0 & 0 & 0 & & 10 & 11 \\
Condition 5: Motor Speed & 31 & 22 & 24 & 10 & 12 \\
\hline
\end{tabular}

D-KEFS scaled scores (in relation to age and total sample): The mean is 10. The standard deviation is 3.8 to 12 is considered average. 7 or lower is considered below average and 13 or higher above average

\section{WAIS-IV processing speed}

The scores on the Processing Speed Index (Table 4) were average compared to the normative population for age at all three test times. There were no relevant differences between time points. She scored 93 at admission $\left(\mathrm{T}_{0}\right)$ and re-test $\left(\mathrm{T}_{1}\right)$ and 98 at follow-up $\left(\mathrm{T}_{2}\right)$.

\section{d2-R test of attention}

At 2 weeks after admission $\left(\mathrm{T}_{0}\right)$ and re-test $\left(\mathrm{T}_{1}\right)$, she had a small number of processed targets (426 and 420), 18th to 21st percentile (Tables 3 and 4), her concentration performance was 175 and 176 corresponding to the 42nd percentile and she committed three and no errors respectively ( $>90$ th percentile). At follow-up $\left(T_{2}\right)$, her concentration performance was above the mean (185; 54th percentile) but not increased more than $S_{\text {diff: }} 24.89$. The total processed targets score was still low $(451 ; 34$ th percentile), and she committed few errors (four; 90th percentile).

\section{Discussion and conclusions}

The patient exhibited average to very superior performance on verbal fluency, design fluency, processing speed, and memory. However, her working memory performance was low average. Her attention and concentration performance were below average to average, and her performance on cognitive flexibility tasks were average to moderately-to-severely impaired.

The present case report demonstrates surprisingly good cognitive performance in a patient with severe and enduring $\mathrm{AN}$ with extremely low BMI varying between 7.7 and 9.3 during the study period of 1 year. However, some of her executive functions seem to be impaired. This is in line with previous research on patients with AN $[5,8]$. The present results suggest that her working memory was normal (low average) in line with previous studies [35, 36]. However, her working memory performance was lower compared to the rest of her memory performance, which was average to very superior. The results from the D-KEFS indicate average to aboveaverage performance with perhaps somewhat weaker cognitive flexibility (below average to average). On the other hand, the results from the WCST indicate impairment in cognitive flexibility. The overall differences in performance between the three assessments were minimal. This indicates that the minor differences in BMI between the test assessments did not significantly affect her cognitive performance, as expected.

\section{Impaired cognitive flexibility}

It could be that impaired cognitive flexibility existed prior to the illness as a premorbid trait as suggested previously [10], or that the malnutrition has affected the patient's cognitive flexibility. Since we are missing data on 
Table 4 The patient's norm scores and percentiles on WMS-III, WAIS-IV Processing Speed Index (PSI), and d2-R at two weeks after admission $\left(T_{0}\right)$, at re-test $\left(T_{1}\right)$, and at follow-up $\left(T_{2}\right)$

\begin{tabular}{|c|c|c|c|c|c|c|c|c|c|}
\hline \multirow[t]{2}{*}{ Instrument } & \multicolumn{3}{|c|}{ Norm scores } & \multicolumn{3}{|c|}{ Percentiles } & \multicolumn{3}{|c|}{$95 \%$ confidence intervals } \\
\hline & $\mathrm{T}_{0}$ & $\mathrm{~T}_{1}$ & $\mathrm{~T}_{2}$ & $\mathrm{~T}_{0}$ & $\mathrm{~T}_{1}$ & $\mathrm{~T}_{2}$ & $\mathrm{~T}_{0}$ & $\mathrm{~T}_{1}$ & $\mathrm{~T}_{2}$ \\
\hline \multicolumn{10}{|l|}{ WMS-III Indexes: } \\
\hline Auditory Immediate & 138 & 138 & 142 & 99 & 99 & 99.7 & $128-143$ & $128-143$ & $132-146$ \\
\hline Auditory Delayed & 132 & 108 & 132 & 98 & 70 & 98 & 119-137 & $98-116$ & 119-137 \\
\hline Visual Immediate & 115 & 118 & 127 & 84 & 88 & 96 & $102-123$ & $105-125$ & $112-132$ \\
\hline Visual Delayed & 118 & 125 & 109 & 88 & 95 & 73 & $105-125$ & $111-131$ & $97-118$ \\
\hline Immediate Memory & 132 & 134 & 142 & 98 & 99 & 99.7 & $121-137$ & $123-139$ & $131-146$ \\
\hline Auditory Recognition Delayed & 120 & 130 & 120 & 91 & 98 & 91 & $104-126$ & $111-133$ & $104-126$ \\
\hline General Memory & 130 & 125 & 125 & 98 & 95 & 95 & 119-135 & $115-131$ & $115-131$ \\
\hline Working Memory & 102 & 88 & 96 & 55 & 21 & 39 & $92-111$ & 80-99 & 98-106 \\
\hline WAIS-IV PSI & 93 & 93 & 98 & 32 & 32 & 45 & 84-104 & 84-104 & 89-108 \\
\hline \multicolumn{10}{|l|}{ d2-R } \\
\hline Processed Targets & & & & 21 & 18 & 34 & & & \\
\hline Errors & & & & $>90$ & 99 & 90 & & & \\
\hline Accuracy (\% errors) & & & & $>90$ & 99 & 90 & & & \\
\hline Corrected total score & & & & 31 & 27 & 42 & & & \\
\hline Concentration Performance & & & & 42 & 42 & 54 & & & \\
\hline
\end{tabular}

WMS-III norm scores (in relation to age): The mean is 100.69 and below is extremely low. 70 to 79 is borderline. 80 to 89 is a low average. 90 to 109 is average. 110 to 119 is a high average. 120 to 129 is superior. 130 and above is very superior. WAIS-IV Processing Speed Index norm score (in relation to age): The mean is 100. 69 and below is extremely low. 70 to 79 is borderline. 80 to 89 is a low average. 90 to 109 is average. 110 to 119 is a high average. 120 to 129 is superior. 130 and above is very superior. $\mathrm{d} 2$ percentiles (in relation to age): The mean is 50

her premorbid level, we cannot draw any firm conclusions.

Impaired cognitive flexibility has previously been reported in patients with AN with higher BMI [37], indicating that impairments in cognitive flexibility do not

Table 5 The patient's scores on the WCST at two weeks after admission $T_{0}$

\begin{tabular}{llcl}
\hline Wisconsin Card Sorting Test & Raw scores & Standard scores & Percentiles \\
\cline { 2 - 4 } Trials administered & 128 & & \\
Correct & 78 & 79 & 8 \\
Errors & 50 & 55 & $<1$ \\
Perseverative Responses & 52 & 65 & 1 \\
Perseverative Errors & 36 & 94 & 34 \\
Non-perseverative Errors & 14 & & \\
Conceptual level responses & 61 & & $<1$ \\
Categories Completed & 1 & & $<1$ \\
Failure to Maintain Set & 4 &
\end{tabular}

WCST standard scores (in relation to years of education): Standard scores greater than three standard deviations below the mean are considered in the severely impaired range (less than or equal to 54 ); 55 to 61 are in the moderately-to-severely impaired range; 62 to 69 are in the moderately impaired range; 70 to 76 are in the mildly-to-moderately impaired range; 77 to 84 are in the mildly impaired range; 85 to 91 are in the below average range; 92 to 106 are in the average range; standard scores equal to or greater than 107 are in the above-average range

Note. The standard score for non-perseverative errors was average since most of her errors were perseverative necessarily relate to undernutrition. In patients with AN who had recovered from the illness, cognitive flexibility was in the normal range in this study. However, other studies found that individuals who recovered from AN exhibited more or less impaired executive functioning [10]. Longitudinal research on the relationship between different BMI states and cognitive performance is highly needed.

Impaired cognitive flexibility may also play a role in the perpetuation of AN. Impaired cognitive flexibility has been suggested as a maintenance factor [38] and a factor related to lack of illness insight characteristic of patients with restrictive AN [39]. Lack of illness insight could be related to treatment resistance [40]. The patient's low scores on EDI-3 subscales also reflect a discrepancy between illness severity and self-reported symptoms. This discrepancy or ambivalence is part of the nature of the disorder reflected in the low motivation for recovery and high number of dropouts from treatment alongside an expressed desire to change [41].

\section{Cognitive adaptation in anorexia nervosa}

Survival of long-term starvation is only possible due to extensive adaptive endocrine and metabolic alterations [42]. How these alterations affect cognitive functions still remains to be clarified. Well-designed longitudinal studies on severely underweight patients with a long illness 
duration are lacking. However, the present case report suggests that essential preservation of some cognitive functions occurs even in extreme chronic semistarvation.

The mechanisms allowing for such preservation remains a subject of speculation. Links can be made to research on neuroplasticity and functional reorganization of cognitive functions after brain injury since patients with AN have white matter alterations [43]. Research shows that brain maturation processes of especially the prefrontal cortex continue until people are approximately 25 years old [44]. Nutritional status seems to impact this brain maturation [44]. Executive functions associated with the prefrontal cortex could therefore be affected by undernutrition during development of prefrontal connections in the brain in adolescence and young adulthood. Thus, impairment on executive functions may not arise until adulthood in patients with AN. This is in line with research that found no cognitive flexibility impairment in children and adolescents with AN but impairments in adults with AN $[19,20]$. The literature indicates that other cognitive functions associated with the prefrontal cortex, such as memory, are also impaired in adults with AN [3]. However, overall, this literature is not as explicit as the literature showing cognitive flexibility impairment in adults with AN. The ambiguity in the literature indicates differences between cognitive functions related to the prefrontal cortex in patients with AN. It might be that some prefrontal connections potentially being affected during low weight in adolescence could be reorganized or "compensated for" with time as is possible with reorganization or apparent functional recovery after brain injury [45]. In that case, cognitive performance could be regained after impairment has occurred. Some dimensions of cognitive flexibility might, however, be more difficult to compensate for. This could explain specific cognitive flexibility impairment in patients recovered from $\mathrm{AN}$ [10] and explain that the patient in the present case report performed normal and superior on some functions associated with prefrontal connections (memory and verbal fluency) but poorer on cognitive flexibility. We therefore suggest that reorganization of some cognitive functions can occur in spite of persisting low weight in patients with AN. In line with the possibility of cognitive reorganization in AN, Cognitive Remediation Therapy seems to improve executive functioning in patients with AN [46]. The suggested theory of cognitive adaptation may therefore not be specific to persisting low weight in AN. However, fast, substantial weight-loss could affect cognitive performance differently than persisting low weight. Therefore, studies on starving healthy subjects, including the Minnesota Semi-Starvation Experiment [16], could show different results than studies on patients with severe and enduring AN. Likewise, studies on patients with short illness duration might find different results than studies of patients with enduring AN. It is also unclear if patients developing $\mathrm{AN}$ in adulthood will display the same cognitive impairments. In line with these reflections, a case report of a 27-year-old Japanese woman in a coma, with BMI of $8.5 \mathrm{~kg} / \mathrm{m}^{2}$ at admission, describes a patient with AN where the outcome of severe malnutrition was persistent neurologic sequelae [47]. The woman developed AN at the age of 21 years where the patient in the present case report was diagnosed at the age of 10 years. The difference in age of onset, duration of illness, and/or manner of weight-loss (fast, substantial weight-loss compared to persisting low weight) may have resulted in different outcomes for the women. It is, however, also a possibility that the patient in the present case report might have an extreme phenotype which enables her to perform well in spite of her being extremely underweight.

We cannot say how high the patient's scores on the neuropsychological test battery might be if she had not been as malnourished. We assume the patient would perform better on cognitive flexibility tasks, that her processing speed and working memory would be higher, and that she would be able to concentrate better had she not been malnourished. This is somewhat supported by previous research. Although the literature suggests impaired cognitive performance in patients with $\mathrm{AN}$, the reported impairments were limited compared to healthy subjects $[8,48]$. Furthermore, it may be that severely underweight patients with AN have a higher verbal IQ [49], which does not, however, exclude the possibility of specific cognitive impairments [50]. This could explain the patient's high memory performance (and probably global IQ) alongside specific impairment in cognitive flexibility on the WCST. This case may therefore not differ from other patients with severe AN regarding cognitive performance. It may be that the superior performance related to some cognitive functions is a trait of severely underweight patients with $\mathrm{AN}$ and/or that a cognitive adaptation to enduring AN increases performance to the premorbid level. In this case, (regained) superior performance of some cognitive functions (i.e. memory and verbal IQ) can exist alongside cognitive impairment in others (i.e. cognitive flexibility). This may change our view of the cognitive profile and its development in patients with severe and enduring AN.

Regardless, the fact that we were able to test the patient in the present case, raises a discussion as to whether she and others with extremely low weight may be responsive to psychotherapy as well. In the present case, the patient underwent psychotherapy for several years albeit without any impact on her weight. More research focusing on the validation of neuropsychological 
tests including investigation of the practice effect in this patient population is needed.

The individual scores on neuropsychological tests should always be interpreted with care. Factors other than persisting low weight may affect neuropsychological performance (e.g. dehydration, stress, depression, and anxiety). In the present case, the patient did express depressive symptoms corresponding to moderate depression, which might have influenced results on impairment in cognitive flexibility. Furthermore, the patient might experience other issues related to cognitive performance in daily life, which cannot be discovered in a neuropsychological assessment context.

Obviously, conclusions can never be drawn from one case. However, since the neuropsychological testing included a broad range of tests and was repeated three times during a year, the present case report is valid as a basis for reflecting on the affected individual's cognitive performance at this stage. The present case report demonstrates that cognitive functions may be largely preserved under extreme chronic malnutrition or that cognitive functioning may be regained (reorganized) in spite of extreme chronic malnutrition. More research on patients with AN with extremely low BMI $(<10)$ is needed to determine whether cognitive performance is affected by starvation and malnutrition.

\section{Abbreviations \\ AN: Anorexia nervosa; IQ: Intelligence quotient; BMI: Body mass index; BDI- II: The Beck Depression Inventory II; EDI-3: The Eating Disorder Inventory 3; WMS-III: The Wechsler Memory Scale III; WAIS-IV: The Wechsler Adult Intelligence Scale IV; PSI: The Processing Speed Index; D-KEFS: The Delis- Kaplan Executive Function System; WCST: The Wisconsin Card Sorting Test Revised and Expanded}

\section{Acknowledgements}

We would like to thank Professor Jesper Mogensen at the Unit for Cognitive Neuroscience, University of Copenhagen, Denmark, for his inputs regarding neurocognitive reorganization and the possibility of extending his model to the research field of anorexia nervosa.

\section{Authors' contributions}

SDH and RKS completed the data collection. RKS was the initiator of the project. SDH, RKS, and MBL all took part in the design of the study. SDH, RKS, $M B L, J M S$ and $A A H$ were all contributors in writing the manuscript. All authors read and approved the final manuscript.

\section{Funding}

The study was supported by government funding: The Psychiatric Research Fund of Southern Denmark (grants for material and PhD salary) and the University of Southern Denmark (faculty scholarship). Furthermore, the study was supported with grants for material by private funds: the Jascha Foundation and the Beckett Foundation. The funding sources had no role in the design, execution, interpretation, analysis, or publication of the study.

\section{Availability of data and materials}

All data analyzed during this study are included in this published article in tables or text. Raw data in a fully anonymized version is available from the corresponding author on reasonable request.

\section{Ethics approval and consent to participate}

The research project has been approved by the Regional Scientific Ethical Committee for the Region of Southern Denmark and was carried out in accordance with the 1964 Helsinki declaration and its later amendments. The authors state that the patient has given written and informed consent for participation in the study.

\section{Consent for publication}

The authors state that the patient has given written and informed consent for publication of the case report.

\section{Competing interests}

The authors declare that there are no conflicts of interest. None of the authors have received financial support or benefits from commercial sources for this study.

\section{Author details}

${ }^{1}$ Centre for Eating Disorder, Odense University Hospital, Odense, Denmark. ${ }^{2}$ Research Unit for Medical Endocrinology, Odense University Hospital, Odense, Denmark. ${ }^{3}$ Department of Clinical Research, University of Southern Denmark, Odense, Denmark. ${ }^{4}$ Open Patient data Explorative Network (OPEN), Odense, Denmark. ${ }^{5}$ The Research Unit, Child and Adolescent Psychiatry, Mental Health Services in the Region of Southern Denmark, Odense, Denmark. ${ }^{6}$ Centre for Telepsychiatry, Mental Health Services in the Region of Southern Denmark, Odense, Denmark. ${ }^{7}$ Department of Psychology, University of Southern Denmark, Odense, Denmark. ${ }^{8}$ Eating Disorder Unit, Mental Health Centre Ballerup, Mental Health Services in the Capital Region of Denmark, Copenhagen, Denmark. ${ }^{9}$ Department of Clinical Medicine, University of Copenhagen, Copenhagen, Denmark.

Received: 13 February 2020 Accepted: 28 May 2020

Published online: 05 June 2020

\section{References}

1. Seed JA, Dixon RA, McCluskey SE, Young AH. Basal activity of the hypothalamic-pituitary-adrenal axis and cognitive function in anorexia nervosa. Eur Arch Psychiatry Clin Neurosci. 2000;250(1):11-5.

2. Seed JA, McCue PM, Wesnes KA, Dahabra S, Young AH. Basal activity of the HPA axis and cognitive function in anorexia nervosa. Int J Neuropsychopharmacol. 2002;5(1):17-25.

3. Biezonski D, Cha J, Steinglass J, Posner J. Evidence for Thalamocortical circuit abnormalities and associated cognitive dysfunctions in underweight individuals with anorexia nervosa. Neuropsychopharmacology. 2016;41(6): 1560-8.

4. Kjaersdam Telleus $G$, Jepsen JR, Bentz M, Christiansen E, Jensen SO, Fagerlund $B$, et al. Cognitive profile of children and adolescents with anorexia nervosa. Eur Eat Disord Rev. 2015;23(1):34-42.

5. Roberts ME, Tchanturia K, Stahl D, Southgate L, Treasure J. A systematic review and meta-analysis of set-shifting ability in eating disorders. Psychol Med. 2007;37(8):1075-84

6. Abbate-Daga G, Buzzichelli S, Marzola E, Aloi M, Amianto F, Fassino S. Does depression matter in neuropsychological performances in anorexia nervosa? A descriptive review. Int J Eat Disord. 2015;48(6):736-45.

7. Bodell LP, Keel PK, Brumm MC, Akubuiro A, Caballero J, Tranel D, et al. Longitudinal examination of decision-making performance in anorexia nervosa: before and after weight restoration. J Psychiatr Res. 2014;56:150-7.

8. Hirst RB, Beard CL, Colby KA, Quittner Z, Mills BM, Lavender JM. Anorexia nervosa and bulimia nervosa: a meta-analysis of executive functioning. Neurosci Biobehav Rev. 2017;83:678-90.

9. Tchanturia K, Davies H, Roberts M, Harrison A, Nakazato M, Schmidt U, et al. Poor cognitive flexibility in eating disorders: examining the evidence using the Wisconsin card sorting task. PLoS One. 2012;7(1):e28331.

10. Tchanturia K, Morris RG, Anderluh MB, Collier DA, Nikolaou V, Treasure J. Set shifting in anorexia nervosa: an examination before and after weight gain, in full recovery and relationship to childhood and adult OCPD traits. J Psychiatr Res. 2004;38(5):545-52.

11. Danner UN, Sanders N, Smeets PA, van Meer F, Adan RA, Hoek HW, et al. Neuropsychological weaknesses in anorexia nervosa: set-shifting, central coherence, and decision making in currently ill and recovered women. Int J Eat Disord. 2012;45(5):685-94.

12. Wu M, Brockmeyer T, Hartmann M, Skunde M, Herzog W, Friederich H-C Reward-related decision making in eating and weight disorders: a systematic review and meta-analysis of the evidence from neuropsychological studies. Neurosci Biobehav Rev. 2016;61:177-96. 
13. Lozano-Serra E, Andres-Perpina S, Lazaro-Garcia L, Castro-Fornieles J. Adolescent anorexia nervosa: cognitive performance after weight recovery. J Psychosom Res. 2014;76(1):6-11.

14. Firk C, Mainz V, Schulte-Ruether M, Fink G, Herpertz-Dahlmann B, Konrad K Implicit sequence learning in juvenile anorexia nervosa: neural mechanisms and the impact of starvation. J Child Psychol Psychiatry. 2015;56(11):1168-76.

15. American Psychiatric Association. Diagnostic and statistical manual of mental disorders. 5th ed. Washington: Author; 2013.

16. Keys A, Brozek J, Henschel A, Mickelsen O, Taylor HL. The biology of human starvation (2 volumes). Minnesota: University of Minnesota Press; 1950.

17. Benau EM, Orloff NC, Janke EA, Serpell L, Timko CA. A systematic review of the effects of experimental fasting on cognition. Appetite. 2014;77:52-61.

18. Buhren K, Mainz V, Herpertz-Dahlmann B, Schafer K, KahramanLanzerath $B$, Lente $C$, et al. Cognitive flexibility in juvenile anorexia nervosa patients before and after weight recovery. J Neural Transm (Vienna). 2012;119(9):1047-57.

19. Telleus GK, Fagerlund B, Jepsen JR, Bentz M, Christiansen E, Valentin JB, et al. Are weight status and cognition associated? An examination of cognitive development in children and adolescents with anorexia nervosa 1 year after first hospitalisation. Eur Eat Disord Rev. 2016;24(5):366-76.

20. Lang K, Stahl D, Espie J, Treasure J, Tchanturia K. Set shifting in children and adolescents with anorexia nervosa: an exploratory systematic review and meta-analysis. Int J Eat Disord. 2014;47(4):394-9.

21. Taylor SE. Adjustment to threatening events: a theory of cognitive adaptation. Am Psychol. 1983;38(11):1161-73.

22. Frolich J, Palm CV, Stoving RK. To the limit of extreme malnutrition. Nutrition. 2016;32(1):146-8

23. Robinson P, Jones WR. MARSIPAN: management of really sick patients with anorexia nervosa. BJPsych Advances. 2018;24(1):20-32.

24. Beck AT, Steer RA, Brown GK. Manual for the Beck depression inventory-II. San Antonio: Psychological Corporation; 1996

25. Garner DM. Eating disorder Inventory-3. Professional manual. Lutz: Psychological Assessment Resources, Inc.; 2004.

26. Clausen L, Rosenvinge $\mathrm{JH}$, Friborg $\mathrm{O}$, Rokkedal K. Validating the eating disorder Inventory-3 (EDI-3): a comparison between 561 female eating disorders patients and 878 females from the general population. J Psychopathol Behav Assess. 2011;33(1):101-10.

27. Wechsler D. WAIS-III WMS-III technical manual. San Antonio: The Psychological Corporation; 2002.

28. Brickenkamp $\mathrm{R}$, Schmidt-Atzert $\mathrm{L}$, Liepmann D. The $\mathrm{d} 2$ test of attention revised. A test of attention and concentration. Oxford: Hogrefe; 2016.

29. Brickenkamp R. d2-testen - en vurdering af opmærksomhed of koncentration. Dansk vejledning. Hogrefe Psykologisk Forlag: Frederiksberg; 2006

30. Wechsler D. Wechsler adult intelligence scale-fourth edition. Technical and interpretive manual. Pearson: San Antonio; 2008.

31. Delis DC, Kaplan E, Kramer JH. Delis-Kaplan executive function system technical manual. Pearson: San Antonio; 2001.

32. Heaton RK, Chelune GJ, Talley JL, Kay GG, Curtiss G. Wisconsin Card Sorting Test Manual Revised and Expanded. 2nd edition ed. Lutz: Psychological Assessment Resources, Inc.; 1993.

33. Tulsky D, Zhu J, Ledbetter M, editors. WAIS-III WMS-III technical manual (Wechsler Adult Intelligence Scale \& Wechsler Memory Scale) paperback updated. USA: The Psychological Corporation; 2002.

34. Iverson GL. Interpreting change on the WAIS-III/WMS-III in clinical samples. Arch Clin Neuropsychol. 2001;16(2):183-91.

35. Bradley SJ, Taylor MJ, Rovet JF, Goldberg E, Hood J, Wachsmuth R, et al. Assessment of brain function in adolescent anorexia nervosa before and after weight gain. J Clin Exp Neuropsychol. 1997;19(1):20-33.

36. Lauer CJ, Gorzewski B, Gerlinghoff M, Backmund H, Zihl J. Neuropsychological assessments before and after treatment in patients with anorexia nervosa and bulimia nervosa. J Psychiatr Res. 1999:33:129-38.

37. Tenconi E, Santonastaso P, Degortes D, Bosello R, Titton F, Mapelli D, et al. Set-shifting abilities, central coherence, and handedness in anorexia nervosa patients, their unaffected siblings and healthy controls: exploring putative endophenotypes. World J Biol Psychiatry. 2010;11(6):813-23.

38. Steinglass JE, Walsh BT, Stern Y. Set shifting deficit in anorexia nervosa. J Int Neuropsychol Soc. 2006;12(3):431-5.

39. Konstantakopoulos G, Tchanturia K, Surguladze SA, David AS. Insight in eating disorders: clinical and cognitive correlates. Psychol Med. 2011:41(9): $1951-61$.
40. Abbate-Daga G, Amianto F, Delsedime N, De-Bacco C, Fassino S. Resistance to treatment and change in anorexia nervosa: a clinical overview. BMC Psychiatry. 2013;13(1):294.

41. Guarda AS. Treatment of anorexia nervosa: insights and obstacles. Physiol Behav. 2008;94(1):113-20.

42. Stoving RK. Mechanisms In Endocrinology: Anorexia nervosa and endocrinology: a clinical update. Eur J Endocrinol. 2019;180(1):R9-r27.

43. Barona M, Brown M, Clark C, Frangou S, White T, Micali N. White matter alterations in anorexia nervosa: evidence from a voxel-based meta-analysis. Neurosci Biobehav Rev. 2019;100:285-95.

44. Arain M, Haque M, Johal $L$, Mathur $P$, Nel W, Rais A, et al. Maturation of the adolescent brain. Neuropsychiatr Dis Treat. 2013;9:449-61.

45. Mogensen J. Reorganization of the injured brain: implications for studies of the neural substrate of cognition. Front Psychol. 2011;2:7.

46. Tchanturia $\mathrm{K}$, Lounes $\mathrm{N}$, Holttum S. Cognitive remediation in anorexia nervosa and related conditions: a systematic review. Eur Eat Disord Rev. 2014;22(6):454-62.

47. Bando N, Watanabe K, Tomotake M, Taniguchi T, Ohmori T. Central pontine myelinolysis associated with a hypoglycemic coma in anorexia nervosa. Gen Hosp Psychiatry. 2005;27(5):372-4

48. Dobson KS, Dozois DJ. Attentional biases in eating disorders: a metaanalytic review of Stroop performance. Clin Psychol Rev. 2004;23(8):1001-22.

49. Schilder CMT, van Elburg AA, Snellen WM, Sternheim LC, Hoek HW, Danner UN. Intellectual functioning of adolescent and adult patients with eating disorders. Int J Eat Disord. 2017;50(5):481-9.

50. Lena SM, Fiocco AJ, Leyenaar JK. The role of cognitive deficits in the development of eating disorders. Neuropsychol Rev. 2004;14(2):99-113.

\section{Publisher's Note}

Springer Nature remains neutral with regard to jurisdictional claims in published maps and institutional affiliations.
Ready to submit your research? Choose BMC and benefit from:

- fast, convenient online submission

- thorough peer review by experienced researchers in your field

- rapid publication on acceptance

- support for research data, including large and complex data types

- gold Open Access which fosters wider collaboration and increased citations

- maximum visibility for your research: over $100 \mathrm{M}$ website views per year

At $\mathrm{BMC}$, research is always in progress.

Learn more biomedcentral.com/submissions 